\title{
Ansatz zur Erarbeitung einer Methodenauswahl für nachhaltige Produktentwicklung in KMUs
}

Björn Ragnar Kokoschko, Laura Augustin, Christiane Beyer, Michael Schabacker

Klein- und mittelständische Unternehmen (KMU) sehen immer mehr den Herausforderungen und Chancen einer nachhaltigen Produktentwicklung entgegen, da die Entwicklung und Herstellung von Produkten zwangsläufig mit Umweltaspekten verbunden sind. Eine gute Anwendung von Methoden und Werkzeugen bestimmt so mitunter über Erfolg und Misserfolg eines Produktes als auch dessen Nachhaltigkeit und ist damit zentrales Element bei der Entwicklungsarbeit. Somit bestimmt eine dauerhafte Integration von Werkzeugen eine langfristige Wettbewerbsfähigkeit von Unternehmen. Da die geringeren Kapazitäten und Rahmenbedingungen von KMUs jedoch eine Barriere bei der Integration von Methoden und Werkzeugen darstellt, wird ein Ansatz beleuchtet, der mit Zuhilfenahme von Filtern bei der passenden Auswahl von Methoden und Werkzeugen Unterstützung bietet. Es werden Hemmschwellen und Erfolgsfaktoren bei der Anwendung von Methoden und Werkzeugen beleuchtet und ein möglicher Prozess skizziert, um die Integration zu fördern, die Mitarbeiter des Unternehmens zu schulen und langfristig die Anwendung von Methoden und Werkzeugen zur nachhaltigen Produktentwicklung in KMUs zu implementieren.

Keywords: Nachhaltigkeitsmethoden, Klein- und mittelständische Unternehmen, Methode

\section{Motivation}

Die Entwicklung und Herstellung von Produkten ist zwangsläufig mit Umweltaspekten verbunden, die zu negativen Umweltauswirkungen führen (Herrmann 2010, 7). Dieses Verständnis der unmittelbaren Auswirkungen von Entwicklung und Herstellung auf die Umwelt und wie Produkte die Ökosysteme um sie herum beeinflussen können, bietet die Möglichkeit, Produkte auf ökologische Anforderungen auszurichten und einen Übergang von einer Gesellschaft der Massenproduktion, des Massenkonsums und der Massenentsorgung zu einer nachhaltigen Gesellschaft, in der Materialien über den gesamten Produktlebenszyklus effizient genutzt werden, zu schaffen (Nakayama et al. 12-14 Dec. 2005, 43). Dies setzt voraus, dass die Produktentwicklung als ein ganzheitlicher und vernetzter Prozess betrachtet wird. Da Produktions- und Umweltprobleme lange Zeit unabhängig voneinander behandelt wurden (Vajna 2014, 315), wurde der 
Umwelt in den Phasen des Produktentwicklungsprozesses wenig Aufmerksamkeit geschenkt (Huang 1996, 1-18). Insbesondere kleine und mittlere Unternehmen (KMU) werden im Vergleich zu Großunternehmen durch geringere Kapazitäten zunehmend vor die Chance gestellt, ihre Innovationsaktivitäten mit den ökologischen Anforderungen an die deutsche Nachhaltigkeits-Strategie (BK-Amt 1. Oktober 2016, Kabinettbeschluss vom 11. Januar 2017) in Einklang zu bringen. Um KMUs konkurrenzfähig zu erhalten, ist die Adaption von nachhaltigen Produktentwicklungsmethoden und Werkzeugen wichtig (O'Connor und Cox 12-14 Dec. 2005). Im Folgenden wird nicht weiter zwischen beiden unterschieden, sondern sie werden der Einfachheit halber unter dem Begriff "Techniken" zusammengefasst. KMUs stellen bisweilen ein Schlüsselelement der Volkswirtschaften auf der ganzen Welt dar (Ministerium für Wirtschaft, Wissenschaft und Digitalisierung des Landes Sachsen-Anhalt 2020), (Hansen et al. 2013), (Masum und Fernandez 2008), (O'Connor und Cox 12-14 Dec. 2005) und spielen eine bedeutende Rolle bei Design, Entwicklung und Herstellung neuer Produkte. Viele dieser Unternehmen haben keinen strukturierten Innovationsprozess, da ihnen Forschungs- und Entwicklungsabteilungen, Budgetierung etc. fehlen (Lindahl 12-14 Dec. 2005). Zudem werden KMU-spezifische Rahmenbedingungen nicht adressiert, wie z. B. geringe Kapazitäten und teilweise fehlende Daten zum Produktlebenszyklus, fehlende Innovations- und Nachhaltigkeitskompetenz, um ökologische Innovationen komplett eigenständig zu entwickeln. Diese müssen jedoch bei der Technikauswahl berücksichtigt werden (Hansen et al. 2013). Dieser Beitrag setzt sich mit den in vorhergehenden Literaturanalysen identifizierten Methoden und den Kriterien für deren Anwendung auseinander und ordnet diese möglichen Produktentwicklungsphasen und Arbeitspaketen zu.

\section{Grundlagen}

Die Vielfalt an Techniken stellt eine Barriere für die Anwendung in der Industrie dar (Pigosso et al. 2015), (Ernzer und Birkhofer 2002, 1305), insbesondere in KMUs. Begrenzte Ressourcen in KMUs führen zu einem Mangel an Wissen über Techniken (Reinicke 2004) und die Anwendung von Techniken (Albers et al. 2014). Selbst wenn das Wissen über die zu wählende Technik vorhanden ist, können Konstrukteure auf das Problem der Kompatibilität einer Technik mit einer anderen stoßen (Ernzer und Birkhofer 2002, 1305). Darüber hinaus basiert die Auswahl der Technik in der Industrie oft auf der Beliebtheit einer Technik und nicht auf einer umfassenden Analyse der Bedürfnisse der Unternehmen (Ritzen und Lindahl 11-15 Dec. 2001), (Guérineau et al. 2018). Zudem ist die Auswahl von Techniken oftmals zufällig und unstrukturiert (Ritzen und 
Lindahl 11-15 Dec. 2001, 3). Dass Techniken in der Industrie nicht angenommen werden, kann auch daran liegen, dass sie nicht auf den entsprechenden Kontext ausgerichtet sind (Petersson und Lundberg 2016).

Um nachhaltige Techniken in KMUs durch eine passgenaue Technikauswahl in verschiedenen Phasen des Produktentstehungsprozesses und unter sich ändernden Bedingungen zu implementieren, wurden in Vorarbeiten Kriterien definiert, um eine Vorauswahl treffen zu können. Dadurch soll sichergestellt werden, dass diese in die Unternehmen integriert werden können, ohne dass dafür spezialisiertes Personal benötigt wird.

Bislang gibt es keinen Ansatz einer integrierten Produktentwicklung, der die ökologischen Anforderungen ausreichend berücksichtigt und mit den Anforderungen von KMUs verbindet, und es fehlt an Ansätzen für eine beschleunigte Anwendung in der Industrie (Pigosso et al. 2015).

Nach PIGossı et al. werden sehr spezifische Wirkungskategorien der Nachhaltigkeit in der Ökodesign-Literatur berücksichtigt, in der eine Vielzahl von Methoden und Werkzeugen veröffentlicht sind (Pigosso et al. 2015). Jedoch wird die auf den Kontext und die Bedürfnisse in den Unternehmen zugeschnittene Auswahl von Methoden nicht oder nur unzureichend berücksichtigt (Johansson 2002), (Pigosso et al. 2015). Hier ergibt sich ein Hindernis bedingt durch die Vielfalt von Methoden und Werkzeugen für die Anwendung in der Industrie, da nicht klar ist, in welchen Situationen welche Techniken mit den entsprechenden Werkzeugen relevant sind. Die daraus resultierende Überforderung des nicht spezialisierten Anwenders durch die Vielzahl von Techniken muss vermieden werden (Ernzer et al.). Diese Integrationshürde soll mit der Entwicklung eines Filterprozesses überbrückt werden, um den Zugang und die Verständlichkeit des Einsatzes von Methoden zu verbessern. Dadurch wird der kontextsensitive Einsatz von Techniken gefördert, welcher erfolgsversprechend ist, in unterschiedlichen Produktentwicklungsansätzen Verwendung zu finden. Kontextsensitiv bedeutet, dass die Auswahl der Methoden zur Bewältigung einer Aufgabe innerhalb des Prozessmodells von der Art des Projektes, dem Entwicklungsstand, den zeitlichen Ressourcen und den verfügbaren Daten abhängt (Wiesner et al. 2019). Eine weitere Hürde bei der Integration von Nachhaltigkeitstechniken in Unternehmen ist, dass diese häufig nur die Nachteile einer Integration sehen, wie die Kosten und ein negatives Öko-Image (Abele et al. 2007, 187), (Grüner et al. 1999). Beidem kann durch eine zielgerichtete Technikauswahl mithilfe von umfassenden Filterkriterien entgegengewirkt werden. 


\section{Filterkriterien}

Ernzer und Birkhofer schlagen einen dreistufigen Ansatz zur Auswahl vor, bei dem Techniken, Unternehmen und das Projekt analysiert werden, um Techniken in das Unternehmen integrieren zu können (Ernzer und Birkhofer 2002). Als Grundregel setzen die Autoren voraus, dass die Techniken für den Konstrukteur geeignet und für die Anwendung ausreichend sind. Dann sollten die Techniken auf ein einheitliches Vorgehensmodell abgebildet werden.

Basierend auf dem Vorschlag eines prozessorientierten Modells (Input, Prozess, Output) aus dem Projekt 'the key to innovation' (Birkhofer et al. 2001) und in vorhergehenden Arbeiten eruierten Kriterien zur Selektion von Techniken (Kokoschko et al. 2021) soll daraus eine Synthese geschaffen werden. Diese ist in Abbildung 1: Filter

Demnach bieten sich für die Gestaltung eines „Tools“ Checkboxen und Drop-Down Menüs an, um die Bandbreite der Informationen zielgerichtet abfragen zu können. Die Reihenfolge der Filterkategorien in Abbildung 1 folgt der sukzessiven Eingrenzung durch die Rahmenbedingungen, so folgen die am stärksten reduzierend wirkenden Filter zum Ende der Abbildung.

Beim Erstellen des web-basierten Werkzeuges ist es zudem wichtig, auf die Art der Darstellung und Kommunikation mit dem Anwender und der daraus resultierenden Lernbarkeit einer Technik zu achten. Ferner wird empfohlen, dabei weiterführende Informationen, Umweltwissen, Ansprechpartner und Experten, aber auch Kooperationsmöglichkeiten mit Hochschulen darzustellen und anzubieten. Neben den Technikinformationen sowie den zu erwartenden Ergebnissen, und Durchführungshinweisen sind für eine bestmögliche Integration von Techniken in KMUs Hilfsmittel wie Checklisten, Erläuterungen und Vorlagen bereitzustellen.

abgebildet und stellt den Ansatz einer auf Auswahlfragen basierenden Möglichkeit dar, Unternehmen und deren Umstände zu avisieren. Aus der Beantwortung der Fragen wird ein passender Technikvorschlag generiert. Dies kann in einem web-basierten Werkzeug erfolgen, in welchem, ähnlich einem Online-Verkaufsportal, Filter hinzugenommen werden. Mit dem Fortschreiten dieser als Abfrage gestalteten Filter dünnen sich die angebotenen Techniken aus, und so entsteht eine auf den Kontext bezogene Auswahl an Techniken. Dabei kann dem Nutzer ein geführter Prozess oder ein ungeführtes Ausfüllen der Filter angeboten werden. Das Ergebnis einer Filterung ist eine Auswahl an Techniken, die den eingegebenen Kriterien entsprechen. Dabei ist es denkbar, Empfehlungen der Nachhaltigkeit, Integrierbarkeit und Anwendbarkeit auf Grundlage von den in vorhergehenden Arbeiten definierten Kriterien für KMUs zu geben. 
Wird eine der vorgeschlagenen Techniken selektiert, werden die benötigten Ressourcen, das Vorgehen und die zu erwartenden Ergebnisse beschrieben.

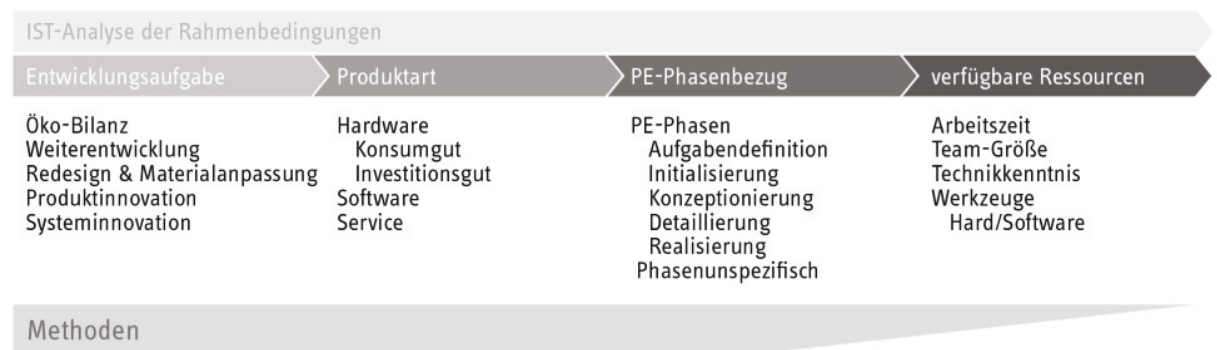

Abbildung 1: Filter

Demnach bieten sich für die Gestaltung eines „Tools“ Checkboxen und Drop-Down Menüs an, um die Bandbreite der Informationen zielgerichtet abfragen zu können. Die Reihenfolge der Filterkategorien in Abbildung 1 folgt der sukzessiven Eingrenzung durch die Rahmenbedingungen, so folgen die am stärksten reduzierend wirkenden Filter zum Ende der Abbildung.

Beim Erstellen des web-basierten Werkzeuges ist es zudem wichtig, auf die Art der Darstellung und Kommunikation mit dem Anwender und der daraus resultierenden Lernbarkeit einer Technik zu achten. Ferner wird empfohlen, dabei weiterführende Informationen, Umweltwissen, Ansprechpartner und Experten, aber auch Kooperationsmöglichkeiten mit Hochschulen darzustellen und anzubieten. Neben den Technikinformationen sowie den zu erwartenden Ergebnissen, und Durchführungshinweisen sind für eine bestmögliche Integration von Techniken in KMUs Hilfsmittel wie Checklisten, Erläuterungen und Vorlagen bereitzustellen.

\section{Entwicklungsaufgabe}

Manch einem Unternehmen ist die konkrete Entwicklungsaufgabe oder deren Schwerpunkt nicht zur Gänze gewahr. Daher soll bei der Auswahlmöglichkeit eine Erläuterung der unterschiedlichen Filter gegeben werden, um nicht spezialisierten KMUs eine fundierte Auswahl zu ermöglichen. Dabei orientieren sich die Unterkategorien an den vier Typen des Öko-Designs nach Brezet und van hemel (Brezet und van Hemel 1997) zzgl. der Erstellung einer Öko-Bilanz (vgl. (Abele et al. 2007)). Z. B. können für eine Material- 
anpassung unterschiedliche Strategien und demnach Techniken als Anwendungsvorschlag in Abhängigkeit der Produktkategorie und der Stückzahl erfolgen. Es kann auch ein ganzheitlicher Prozess vorgeschlagen werden, wenn es sich um eine Produkt- oder Systeminnovation handelt. Wenn jedoch zum Beispiel nicht klar ist, welche Entwicklungsaufgabe bestritten werden soll oder wird, kann die Produktart einer näheren Betrachtung unterzogen werden.

\section{Produktart}

Durch die Auswahl Produktkategorie des durch das Unternehmen zu produzieren Produkts lässt sich der Technikvorschlagkatalog in Anbetracht der für diese Kategorie zutreffenden Techniken reduzieren. Dabei können Mischformen aus den Unterpunkten, also den Produktkategorien, entstehen. Zu der Abfrage einer Produktkategorie kann die Stückzahl und auch die zu verwendenden Materialien, falls diese bereits bekannt sind, Aufschluss über anwendbare Techniken geben.

\section{Produktentwicklungsphasen}

Ein Unternehmen, welches ohne Phasenmodell, also sequentiell abfolgenden Arbeitsschritten mit teilweise zugeordneten Techniken arbeitet, wird durch einen erläuternden Text aufgeklärt. Möglicherweise bietet sich an dieser Stelle eine Entwicklungsstandsabfrage besser als eine Zuordnung zu einer Produktentwicklungsphase. Wenn beispielsweise die Entwicklungsaufgabe klar ist, die Rahmenbedingungen bekannt sind und demnach eine Technik gesucht wird, mit der Ideen generiert werden können, ließen sich die meisten Kreativitätstechniken durch die Auswahl der Konzeptionierung ermitteln.

\section{Verfügbare Ressourcen}

Die verfügbaren Ressourcen haben sich, wie oben beschrieben, als einer der Hauptausschlusspunkte für die Anwendbarkeit von Techniken in KMUs erwiesen. Dabei kann die Technikkenntnisabfrage mögliche synergetische Verknüpfungen aufzeigen, die die Erlernbarkeit einer Technik erleichtern kann; wenn bereits ähnliche Techniken im Unternehmen verwendet werden wie z. B. House of Quality und House of Environment. Die für die Anwendung einer Technik geschätzt verfügbare Arbeitszeit reduziert den Katalog der anwendbaren Techniken und gibt eine Übersicht über die geschätzte Dauer von Techniken. Eine Eingrenzung durch vorhandene Arbeitsmittel wie z. B. Computer, 3D-Drucker oder auch Software kann auch hilfreich sein, wenn es um die schnelle oder interne Umsetzung von Aufgaben geht. Dabei sollte jedoch die Möglichkeit des Einkaufs von Dienstleistungen nicht vergessen werden. Eine interdisziplinäre 
Teamzusammensetzung kann neben einer guten Integration und Umsetzung von Techniken zudem den anwendbaren Technikkatalog erweitern.

\section{Zusammenfassung und Ausblick}

Dieser Beitrag beschreibt eine übertragbare Vorgehensweise, um eine nutzerbasierte Filterung von Methoden und Werkzeugen in der nachhaltigen Produktentwicklung durchzuführen. Auf dieser Grundlage wird ein web-basiertes Werkzeug entwickelt werden, welches Techniken der Nachhaltigkeitsaspekte integriert und mittels Filter selektieren hilft. Dadurch kann ein Verständnis für die Bedürfnisse des Unternehmens geschaffen werden und die Planung und Steuerung von Nachhaltigkeitsaspekten in Unternehmen vorangebracht werden. Zudem kann die Schulung von Personal unterstützt werden, was nach Nach Pigosso, Rozenfeld, McAloone und Tim ein Erfolgsfaktor bei der Technikintegration darstellt (Pigosso et al. 2015, 413). Darüber hinaus wird durch das Vermitteln der Techniken der Mitarbeiter eingebunden und im Idealfall von der Anwendung einer Technik überzeugt; folgend kann eine Technikintegration zur Verankerung dieser bei erfolgreicher Durchführung erfolgen. Ergänzend wird durch die Ausgabe der zu erwartenden Ergebnisse, der Durchführung und der Selektion eines Technikvorschlags ein Bewusstsein für die Methodenanwendung geschaffen. Ferner kann dadurch auch untersucht werden, welches Technikverständnis bei den KMUs gegeben ist, um dann gezielt mit einer Anpassung des Konzeptes oder sogar Workshops antworten zu können. Ideal wäre ein Anpassen der Methoden an die Bedürfnisse der Anwender (Abele et al. 2008, 62).

In Projektarbeiten und Kooperationen mit KMUs werden Chancen und Schwierigkeiten der Anwendbarkeit und Integrierbarkeit betrachtet, die auf Entwicklungspotentiale der Methoden und Werkzeuge hinweisen können. Somit kann eine kontinuierliche Verbesserung der "Techniken" und des Wissens über deren Anwendung erreicht werden, um eine weiterführende, nachhaltige Entwicklung zu fördern.

\section{Literaturverzeichnis}

Abele, Eberhard/Anderl, Reiner/Birkhofer, Herbert (2007). Environmentally-Friendly Product Development. Methods and Tools. Dordrecht, Springer.

Abele, Eberhard/Anderl, Reiner/Birkhofer, Herbert/Rüttinger, Bruno (2008). EcoDesign.

Von der Theorie in die Praxis. Berlin, Heidelberg, Springer-Verlag Berlin Heidelberg.

Albers, Albert/Lüdcke, Robert/Bursac, Nikola/Reiss, Nicolas (2014). Connecting knowledge-Management-Systems to improve a continuous flow of knowledge in engineering design processes. https://doi.org/10.13140/2.1.4612.9447. 
BK-Amt (2016, Kabinettbeschluss vom 2017). Deutsche Nachhaltigkeitsstrategie. Neuauflage 2016. Presse- und Informationsamt der Bundesregierung. 11044 Berlin, Pressemitteilung vom 01.10.2016, Kabinettbeschluss vom 11.01.2017.

Brezet, H./van Hemel, Carolien (Hg.) (1997). Ecodesign.

A promising approach to sustainable production and consumption. The Hague.

Ernzer, M./Birkhofer, H. (2002). Selecting methods for life cycle design based on the needs of a company. In: DS 30: proceedings of DESIGN 2002, the 7th international design conference, Dubrovnik, 1305-1310.

Ernzer, Marc/Oberender, C./Birkhofer, Herbert. DFE for companies - an easy-to-use individualized method mix for DFE. In: Proceedings of ICED 2001.

Guérineau, Benjamin/Rivest, Louis/Bricogne, Matthieu/Durupt, Alexandre/Eynard, Benoît (2018). TOWARDS A DESIGNMETHOD SELECTION FRAMEWORK FOR MULTIDISCIPLINARY PRODUCT DEVELOPMENT. In: Proceedings of the DESIGN 2018 15th International Design Conference, 15th International Design Conference, May, 21-24, 2018. Faculty of Mechanical Engineering and Naval Architecture, University of Zagreb, Croatia; The Design Society, Glasgow, UK, 2879-2890.

Hansen, Erik G./Klewitz, Johanna/Schaltegger, Stefan (2013).

Industrie Management 5/2013: Eco-Innovation. GITO mbH Verlag.

Herrmann, Christoph (2010). Ganzheitliches Life Cycle Management.

Nachhaltigkeit und Lebenszyklusorientierung in Unternehmen. Berlin, Springer.

Huang, G. Q. (1996). Design for X. Dordrecht, Springer Netherlands.

Johansson, Glenn (2002). Success factors for integration of ecodesign in product development. Environmental Management and Health 13 (1), 98-107. https://doi.org/10.1108/09566160210417868.

Kokoschko, Björn/Augustin, Laura/Schabacker, Michael (2021).

Identifying criteria for the selection of ecodesign methods to integrate into SMEs product development 2021.

Lindahl, M. (2005). Designers' Utilization of and Requirements on Design for Environment (DfE) Methods and Tools. In: 2005 4th International Symposium on Environmentally Conscious Design and Inverse Manufacturing, 2005 4th International Symposium on Environmentally Conscious Design and Inverse Manufacturing, Tokyo, Japan, 12-14 Dec. 2005. IEEE, 224231.

Masum, Mohibul Islam/Fernandez, Alejandra (2008). Internationalization Process of SMES:

Strategies and Methods. Master Thesis.

Ministerium für Wirtschaft, Wissenschaft und Digitalisierung des Landes Sachsen-Anhalt (2020). Mittelstandsoffensive Sachsen-Anhalt $\left.\right|^{3}$ - Wachstum durch Innovationen, Investitionen und Internationalisierung. Hier ist der Mittelstand. Online verfügbar unter https://mw.sachsen-anhalt.de/fileadmin/Bibliothek/Politik_und_Verwaltung/MW/Publikationen/Mittelstandsoffensive_Sachsen-Anhalt.pdf (abgerufen am 19.05.2020).

Nakayama, H./Nishino, N./Oda, S. H./Ueda, K. (2005). Decision Making of Economic Agents for Durable-Goods Recycling. In: 2005 4th International Symposium on Environmentally Conscious Design and Inverse Manufacturing, 2005 4th International Symposium on Environmentally Conscious Design and Inverse Manufacturing, Tokyo, Japan, 12-14 Dec. 2005. IEEE, 43-50. 
O'Connor, F./Cox, I. (2005). Ecodesign and Sustainable Business Practice: Insights from design-led SMEs in Wales (UK). In: 2005 4th International Symposium on Environmentally Conscious Design and Inverse Manufacturing, 2005 4th International Symposium on Environmentally Conscious Design and Inverse Manufacturing, Tokyo, Japan, 12-14 Dec. 2005. IEEE, 28-29.

Petersson, Anna Malou/Lundberg, Jan (2016). Applying Action Design Research (ADR) to Develop Concept Generation and Selection Methods. Procedia CIRP 50, 222-227. https://doi.org/10.1016/j.procir.2016.05.024.

Pigosso, Danialea Christina Antelmi/Rozenfeld, Henrique/McAloone, Tim, C. (2015). Characterization of the State-of-the-art and Identification of Main Trends for Ecodesign Tools and Methods. Classifying Three Decades of Research and Implementation. Journal of the Indian Institute of Science 2015 (VOL 95:4), 405-426.

Reinicke, Tamara (2004). Möglichkeiten und Grenzen der Nutzerintegration in der Produktentwicklung. Zugl.: Berlin, Techn. Univ., Diss., 2004. München, Verl. Dr. Hut.

Ritzen, S./Lindahl, M. (2001). Selection and implementation - key activities to successful use of EcoDesign tools. In: Proceedings Second International Symposium on Environmentally Conscious Design and Inverse Manufacturing, Second International Symposium on Environmentally Conscious Design and Inverse Manufacturing, Tokyo, Japan, 11-15 Dec. 2001. IEEE Comput. Soc, 174-179.

Vajna, Sándor (Hg.) (2014). Integrated Design Engineering.

Ein interdisziplinäres Modell für die ganzheitliche Produktentwicklung. Berlin, Springer Vieweg.

Wiesner, Martin/Kokoschko, Björn/Bùi Duy, Linh/Augustin, Laura (2019). Card Sorting basierter Ansatz zur Erarbeitung einer nutzungsgerechten Methodensammlung am Beispiel des IDE-Toolkits. In: Jens Krzywinski/Mario Linke/Christian Wölfel (Hg.). Entwerfen Entwickeln Erleben 2019. eee2019. Dresden, 29-40.

\section{Kontakt}

Björn Ragnar Kokoschko, M. A.

Laura Augustin, M. Sc.

Univ.-Prof. Dr.-Ing. Christiane Beyer

Dr.-Ing. Dipl.-Math. Michael Schabacker

Otto-von-Guericke-Universität Magdeburg

Universitätsplatz 2

39106 Magdeburg

https://ide.ovgu.de 